\title{
Structural and electronic properties of ZnSe/AlAs heterostructures
}

\author{
S. Rubini, E. Milocco, L. Sorba, ${ }^{*}$ E. Pelucchi, and A. Franciosi ${ }^{\dagger}$ \\ Laboratorio Nazionale TASC-INFM, Area Science Park, S.S. 14, Km. 163.5, I-34012 Trieste, Italy \\ A. Garulli and A. Parisini \\ Istituto LAMEL del C.N.R., Via dei Castagnoli 1, I-40126 Bologna, Italy \\ Y. Zhuang and G. Bauer \\ Institut fur Halbleiterphysik, Johannes Kepler Universität Linz, Altenbergerstraße 69, A-4040 Linz, Austria
}

(Received 4 August 2000; published 29 March 2001)

\begin{abstract}
Pseudomorphic ZnSe/AlAs(001) heterostructures were fabricated by molecular beam epitaxy on GaAs wafers. Intrinsic stacking faults on $\{111\}$ planes originating at the II-VI/III-V interface and propagating throughout the II-VI overlayer were the main type of native defects observed. The interface termination was varied by adsorption of $\mathrm{Zn}$ or Se onto the AlAs(001) $3 \times 1$ surface prior to ZnSe growth. The resulting large changes in interface composition and band discontinuities mirror those obtained by employing Zn- or Se-rich growth conditions in the early stages of heterojunction fabrication. Band offsets calculated from first principles for $\mathrm{ZnSe} / \mathrm{GaAs}$, when rescaled by the different magnitude of the electrostatic interface dipole, yield a range of predictions in good agreement with experiment for ZnSe/AlAs.
\end{abstract}

DOI: 10.1103/PhysRevB.63.155312

PACS number(s): 73.40.Lq, 81.15.Hi, 68.35.Ct, 61.72.Nn

\section{INTRODUCTION}

Semiconductor heterojunctions remain challenging systems, as they continue to test our theoretical understanding of the physics of interfaces, and limit the performance of a variety of solid-state devices. The possibility of controlling the electronic properties of semiconductor heterojunctions through modifications of the local interface environment has attracted attention since the very inception of modern interface science. ${ }^{1-3}$

Until very recently, the largest success in this area was the reported tunability of the $\mathrm{ZnSe} / \mathrm{GaAs}(001)$ valence-band offset in the $0.6-1.2 \mathrm{eV}$ range as a result of changes in the local interface composition. ${ }^{4,5}$ Such changes were mostly implemented ${ }^{4-7}$ by employing Se-rich or Zn-rich growth conditions during the early stages of interface fabrication by molecular beam epitaxy (MBE). Early photoemission spectroscopy results obtained in situ on thin-overlayer samples ${ }^{4-7}$ were later systematically confirmed in fully functional heterojunction diodes by internal photoemission ${ }^{8}$ and lowtemperature transport measurements. ${ }^{9,10}$

These early experimental investigations were guided $a$ priori and put into perspective a posteriori by the parallel development of a whole new theoretical framework for semiconductor heterojunctions. The convergence of different theoretical models, including first-principles calculations, the theoretical alchemy approach, ${ }^{11}$ and linear response theory (LRT) results, ${ }^{12,13}$ yielded a unified picture of semiconductor heterojunctions. ${ }^{3,14}$ For isovalent heterojunctions, and for heterovalent heterojunctions with nonpolar orientation, the band alignment would be expected to be, to the first order, a bulk property of the two semiconductor constituents. For such systems charge neutrality is compatible with ideally abrupt interfaces, so that there is little or no enthalpic driving force toward atomic mixing. The bulk-related nature of the band alignment, however, would hold even if mixing were to take place, provided that no cation-anion swaps occur across the interface.

Conversely, ideally abrupt, unreconstructed heterovalent interfaces with a polar orientation would be charged, so that there is a strong, enthalpic driving force toward atomic intermixing. Neutral interfaces can form, lowering the total energy of the system, through a variety of different atomicscale reconstructions at the interface. Consequently, the electronic structure depends on the details of the local atomic structure, and a whole range of band alignments might be possible, depending on the interface configurations achievable in practice. For $\mathrm{ZnSe} / \mathrm{GaAs}(001)$, for example, valenceband offsets in the $0.6-1.6 \mathrm{eV}$ range were predicted based on LRT and self-consistent calculations. ${ }^{4,15}$

The remarkable success achieved by theory in identifying heterovalent heterojunctions as the most promising tunable interface systems, and providing a microscopic rationale for the observed tunability of the offsets, overshadowed the fact that the useful systematics was-and still is-exceedingly scarce. Few correlated experimental and theoretical investigations on the same epitaxial interface system have been performed and often no parallel compositional and structural analysis of the interface was available. More recent results do not always support the conceptual framework described above.

For example, $\mathrm{ZnSe} / \mathrm{GaAs}(001)$ heterojunctions fabricated by metalorganic chemical vapor deposition (MOCVD), predosing the surface with a Se or $\mathrm{Zn}$ precursor prior to $\mathrm{ZnSe}$ growth, did yield valence-band offsets tunable throughout the $0.6-1.1 \mathrm{eV}$ range, ${ }^{16}$ but for reported interface compositions that have little to do with those observed in the MBE studies. ${ }^{4,5,7}$ Also, experimental valence-band discontinuities of 0.46 and $1.26 \mathrm{eV}$ have been reported for device-grade BeTe/ZnSe heterostructures fabricated by $\mathrm{MBE}$ on $\mathrm{Zn}$ stabilized $\mathrm{ZnSe}(001) c(2 \times 2)$ and Se-terminated $\mathrm{ZnSe}(001) 2 \times 1$ surfaces, respectively. ${ }^{17,18}$ This wide offset 
tunability, among the largest reported to date, ${ }^{19}$ is to be considered largely unexpected for an isovalent, as opposed to a heterovalent heterojunction.

In view of the above considerations, we deemed it necessary and timely to expand the available systematics relating growth parameters, interface composition, experimental band alignment, and theoretical predictions for heterovalent heterojunctions. We elected to focus on $\mathrm{ZnSe} / \mathrm{AlAs}(001)$ heterojunctions mostly in view of the many useful similarities and important differences of this interface system as compared to $\mathrm{ZnSe} / \mathrm{GaAs}(001)$.

For example, the lattice mismatch between $\mathrm{ZnSe}$ and AlAs $(0.10 \%)$ is even smaller than that which exists between $\mathrm{ZnSe}$ and GaAs $(0.27 \%)$, minimizing the expected influence of residual strain and lattice relaxation on the results. The chemical similarity between AlAs and GaAs enabled us to simply derive theoretical predictions for $\mathrm{ZnSe} / \mathrm{AlAs}$ from the self-consistent results for $\mathrm{ZnSe} / \mathrm{GaAs}$ through LRT arguments.

On the other hand, the stability of the Al-As bonds is enhanced relative to Ga-As bonds and correspondingly the bandgap increases substantially. This allowed us to investigate if interface chemistry is modified by substrate stability, ascertain how the increased bandgap difference is partitioned between valence and conduction bands, and probe the transitivity of the band offsets.

Last but not least, it has been shown that $\mathrm{ZnSe}$ epilayers can be fabricated by MBE on AlAs buffer layers and $\operatorname{GaAs}(001)$ wafers at growth temperature and for growth rates similar to those normally employed during $\mathrm{ZnSe}$ growth on $\operatorname{GaAs}(001){ }^{20,21}$ Entropic driving forces should therefore play a similar role, if any, for the two interfaces.

\section{EXPERIMENTAL DETAILS}

All epilayers were fabricated by solid-source MBE on $\operatorname{GaAs}(001)$ wafers in a ultrahigh-vacuum facility that includes chambers for III-V and II-VI semiconductor epitaxy, an analysis chamber for in situ monochromatic X-ray photoemission spectroscopy (XPS), and a metallization chamber for in situ contact fabrication.

Following thermal desorption of the native oxide, 0.5$\mu \mathrm{m}$-thick GaAs buffer layers were grown at $600^{\circ} \mathrm{C}$, with a typical As beam equivalent pressure in the $7 \times 10^{-6}$ Torr range, as determined by means of an ion gauge positioned at the sample location, and a Ga to As beam equivalent pressure ratio (BPR) of 1 to $15-20$. Such growth conditions resulted in an As-stabilized $2 \times 4$ reconstruction during growth, as determined by reflection high energy electron diffraction (RHEED) and growth rates in the $1 \mu \mathrm{m} / \mathrm{h}$ range.

AlAs epilayers were then grown on thin $\mathrm{Al}_{0.3} \mathrm{Ga}_{0.7} \mathrm{As}$ buffer layers at $600^{\circ} \mathrm{C}$, with a typical As beam equivalent pressure in the $7 \times 10^{-6}$ Torr range and Al:As BPR of 1:70, resulting in As-stabilized $3 \times 1$ or $3 \times 2$ reconstructions, ${ }^{22}$ and growth rates in the $0.4 \mu \mathrm{m} / \mathrm{h}$ range. As a rule, the AlAs epilayers were protected with an amorphous As cap layer, which was thermally desorbed in the II-VI growth chamber to yield $\mathrm{AlAs}(001) 3 \times 1$ surfaces immediately prior to $\mathrm{ZnSe}$ deposition.
ZnSe epilayers were grown at $290^{\circ} \mathrm{C}$ on the III-V substrates, using elemental $\mathrm{Zn}$ and Se diffusion cells, with individual beam equivalent pressures in the $0.6-6 \times 10^{-6}$ Torr range, and selecting different $\mathrm{Zn}$ :Se BPR's throughout the $0.1-10$ range. Typical growth rates were between 0.2 and 0.3 $\mu \mathrm{m} / \mathrm{h}$.

For the III-V side of the heterostructure we explored different types of doping namely, $n$-type (Si-doped, $n$ $\sim 10^{18} \mathrm{~cm}^{-3}$ ), $p$-type (Be-doped, $p \sim 10^{16}-10^{18} \mathrm{~cm}^{-3}$ ), and nominally undoped (background doping, $p \sim 10^{15} \mathrm{~cm}^{-3}$ ). All $\mathrm{ZnSe}$ epilayers were nominally undoped (background doping, $n \sim 10^{15} \mathrm{~cm}^{-3}$ ).

To modify the composition of the interface, we employed two different methods. The first method used high or low BPR for the fabrication of the first $2 \mathrm{~nm}$ of the II-VI overlayer. Epilayers grown with $\mathrm{BPR} \geqslant 1$ displayed a $c(2 \times 2)$ RHEED pattern, while for $\mathrm{PBR}<1$ a $2 \times 1$ reconstruction was observed. ${ }^{23,24}$ For photoemission determinations of the offset the whole II-VI overlayer was $\sim 2 \mathrm{~nm}$ thick, and therefore grown entirely in nonstoichiometric growth conditions. When thicker overlayers were produced, such as for structural characterization, the rest of the $\mathrm{ZnSe}$ was fabricated with $\mathrm{BPR} \sim 1$. The goal was to implement the type of composition-control interface layer that has proven successful in controlling the band offsets in $\mathrm{ZnSe} / \mathrm{GaAs}$ heterostructures and allowing independent optimization of the structural and optical properties. ${ }^{5,25-27}$

The second method employed to vary the interface composition involved predosing the AlAs surface with an elemental Se or $\mathrm{Zn}$ flux prior to growth of the $\mathrm{ZnSe}$ overlayer with $\mathrm{BPR} \sim 1$. This procedure was performed at the $\mathrm{ZnSe}$ growth temperature (GT) of $290^{\circ} \mathrm{C}$ or at room temperature (RT), with substantially different results. The $\mathrm{Zn}$ and $\mathrm{Se}$ beam equivalent pressure used for predosing the surface, as well as for the subsequent $\mathrm{ZnSe}$ growth with $\mathrm{BPR} \sim 1$ was $1.6 \times 10^{-6}$ Torr.

In general, GT predosing resulted in lower elemental Zn or Se condensation and more ordered surfaces as compared to RT predosing. Exposure of the clean AlAs surface to a $\mathrm{Zn}$ flux at $290^{\circ} \mathrm{C}$ resulted in the gradual removal of the the 3 $\times$ periodicity, and convergence to a streaky $1 \times 1$ RHEED pattern for exposure times longer than $400 \mathrm{~s}$. ZnSe overgrowth at $290^{\circ} \mathrm{C}$ with $\mathrm{BPR}=1$ resulted in a streaky $c(2$ $\times 2)$ RHEED pattern. During exposure of the $\operatorname{AlAs}(3 \times 1)$ surface to Se at $290^{\circ} \mathrm{C}$ the RHEED pattern changed in a few seconds to a $2 \times 1$ reconstruction. No other changes were observed for exposures as long as $600 \mathrm{~s}$. ZnSe growth on the Se-dosed surface was characterized by a spotty RHEED pattern, indicative of tridimensional (3D) growth.

Conversely, RT predosing resulted in higher $\mathrm{Zn}$ or $\mathrm{Se}$ condensation and loss of long-range order, that could be recovered in part through postgrowth annealing. Multilayer $\mathrm{Zn}$ and Se adsorption yielded RHEED patterns involving a superposition of lines and circles, indicative of a partly polycrystalline growth. The $\mathrm{Zn}$ and Se coverage was determined through XPS, as described in the next section. Polycrystalline $\mathrm{ZnSe}$ layers $(\sim 2 \mathrm{~nm}$ thick) were deposited at $\mathrm{RT}$ on AlAs surfaces with one monolayer (ML, in terms of $\mathrm{ZnSe}$ density and lattice parameter, $1 \mathrm{ML}=6.22 \mathrm{at} / \mathrm{cm}^{2}=0.142 \mathrm{~nm}$ ) 


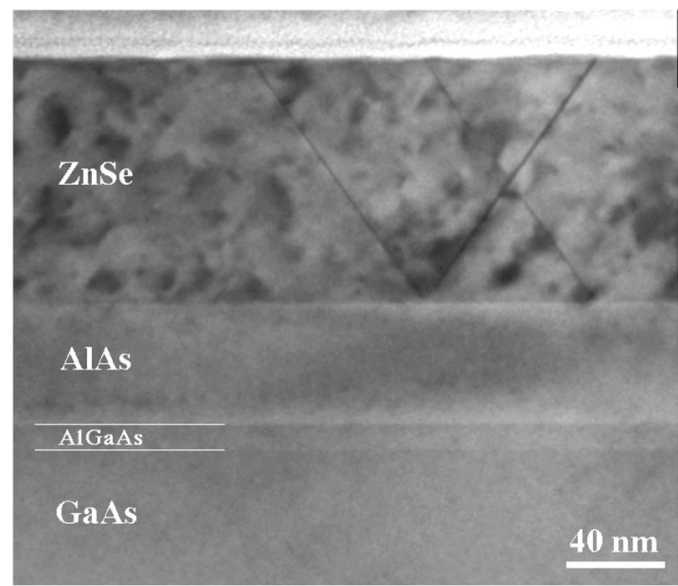

FIG. 1. Bright-field transmission electron microscopy (TEM) cross section of a $\mathrm{ZnSe} / \mathrm{AlAs} / \mathrm{Al}_{x} \mathrm{Ga}_{1-x} \mathrm{As} / \mathrm{GaAs}$ heterostructure. The characteristic $\mathrm{V}$-shaped contrast in the rightmost section of the figure reflects the presence of stacking faults on $\{111\}$ planes.

of adsorbed $\mathrm{Zn}$ or 1-2 ML of adsorbed Se. A postgrowth anneal of $600 \mathrm{~s}$ at $290{ }^{\circ} \mathrm{C}$ was performed to improve crystallinity and achieve a well-defined, but somewhat spotty 1 $\times 1$ RHEED pattern.

XPS measurements were performed in situ using $\mathrm{Al} K \alpha$ $(1486.6 \mathrm{eV})$ radiation monochromatized and focused by a bent crystal monochromator, and a hemispherical electrostatic electron energy analyzer with an overall energy resolution (electrons plus photons) of $0.8 \mathrm{eV}$. The effective escape depth was $1.5 \mathrm{~nm}^{28}$

Structural characterization was performed through $\mathrm{X}$-ray diffraction (XRD) and transmission electron microscopy (TEM) analyses of selected heterostructures. Rocking curves and reciprocal-space maps were recorded using a highresolution diffractometer equipped with a $\mathrm{Cu}$ radiation source and a Bartels 1-crystal Ge(220) monochromatorproviding $\mathrm{Cu} K \alpha_{1}$, radiation $(\lambda=0.154056 \mathrm{~nm})$ with a 12 arc sec angular divergence-and a channel-cut two-crystal $\mathrm{Ge}(220)$ analyzer. ${ }^{29}$ Cross-sectional TEM studies were performed using a Philips CM30-T microscope operated at 300 $\mathrm{kV}$ and equipped with a Gatan $794 \mathrm{MSC}$ camera. Specimens were cut and prepared by conventional ion thinning.

\section{RESULTS AND DISCUSSION}

\section{A. Structure}

An illustration of the main elements of the type of heterostructures employed can be found in the cross-sectional TEM micrograph in Fig. 1. In the bright-field image, markers show the boundaries of the bottom GaAs epilayer, thin $\mathrm{Al}_{0.3} \mathrm{Ga}_{0.7} \mathrm{As}$ buffer (nominal thickness $10 \mathrm{~nm}$ vs an experimental value of $11 \pm 1 \mathrm{~nm}$ in Fig. 1), AlAs epilayer (50 nm vs $49 \pm 3 \mathrm{~nm}$ ), and ZnSe overlayer (100 nm vs $99 \pm 7 \mathrm{~nm}$ ). No extended defects were observed in the heterostructure, but for the stacking faults on $\{111\}$ planes of the II-VI epilayer, giving rise to the characteristic $\mathrm{V}$-shaped contrast in the rightmost section of Fig. 1 .
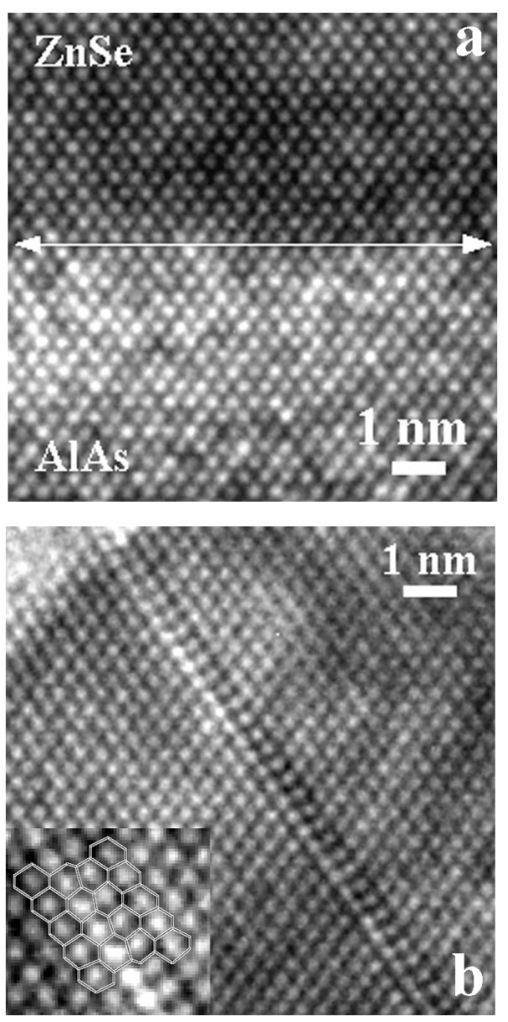

FIG. 2. (a) High-resolution TEM image of a region of the $\mathrm{ZnSe} /$ AlAs interface. (b) High-resolution TEM image recorded near one of the $\{111\}$ stacking fault planes. In the inset, we show an enlarged view with a superimposed schematic of the defect structure.

In Figs. 2(a) and 2(b) we show $\langle 110\rangle$ high-resolution TEM images of regions of the heterostructure near the $\mathrm{ZnSe} /$ AlAs interface and near one of the $\{111\}$ stacking fault planes, respectively. Interatomic channels correspond to the bright regions in the lattice fringes of Fig. 2. The average position of the interface, marked by an arrowed line in Fig. 2(a), has been determined through a vertical intensity line profile averaged over the image width. Finer details of the interface structure are not resolved, for this system, in this crystallographic projection. However, no evidence of misfit dislocations or second phases was found in any of the samples examined.

The stacking faults in Figs. 1 and 2(b) originate at the II-VI/III-V interface and propagate throughout the II-VI epilayers. To our knowledge, such defects have never been reported for the $\mathrm{ZnSe/AlAs}$ system, although stacking faults with similar apparent characteristics are observed in $\mathrm{ZnSe} /$ GaAs heterostructures, and in all II-VI blue-green optoelectronic devices grown to date on GaAs wafers. ${ }^{30}$ Such defects are neither affected by strain, nor do they contribute efficiently to strain relaxation, but play an important role in II-VI-device degradation, ${ }^{30}$ so that they have been attracting substantial interest in recent years.

From Fig. 2(b), it is possible to determine the intrinsic or extrinsic nature of the fault. An enlarged view of a defect portion is shown in the inset of Fig. 2(b). The stacking of double $\{111\}$ planes, ...ABC..., typical of the perfect crystal is seen to change to ...ABA... at the fault location. The latter 


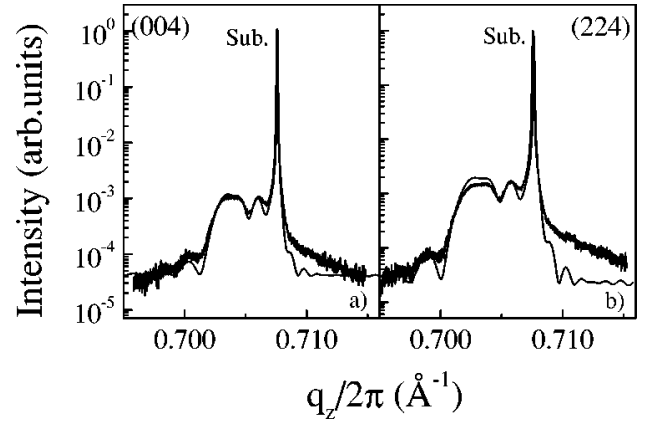

FIG. 3. Experimental rocking curves near the (004) and (224) Bragg reflections [(a) and (b), respectively] are compared with dynamical simulations that assume ideal pseudomorphic growth of ZnSe and AlAs.

stacking sequence is characteristic of intrinsic stacking faults. In the inset, to the enlarged view of the defect we have also superimposed a schematic drawing of the stacking fault structure where the so-called twin units $T$ are marked. From our TEM observations, intrinsic stacking faults appear as the dominant type of defects. This finding is at variance with the results of previous TEM investigations of the $\mathrm{ZnSe} / \mathrm{GaAs}$ heterostructures where extrinsic stacking faults were determined to be the dominant type of defects. ${ }^{31}$ At present, the origin of this difference between $\mathrm{ZnSe} / \mathrm{AlAs}$ and $\mathrm{ZnSe} / \mathrm{GaAs}$ heterostructures is unclear, but its potential impact on device degradation will need to be evaluated.

Although the microscopic mechanism leading to stacking fault nucleation at the II-VI/III-V interface is still controversial, ${ }^{32,33}$ at least some of the models propose strain $^{34,35}$ or the formation of interface reaction products ${ }^{36}$ as major culprits. In view of our TEM results, neither the change in strain nor the change in local chemistry brought about by the replacement of GaAs with AlAs show promise of eliminating the stacking faults issue.

The absence of misfit dislocations in Figs. 1-3 suggests that the whole structure grows pseudomorphically on the GaAs substrate. To gain further insight into the strain status of the different epilayers, ${ }^{37}$ we performed XRD measurements on samples qualitatively similar to those depicted in Fig. $1 .^{38}$

Figures 3 and 4 show, for example, rocking curves and reciprocal space maps, respectively, near the symmetric (004) and the asymmetric (224) and (335) reflections from $\mathrm{ZnSe}$ and AlAs. Rocking-curve simulations were also performed using x-ray dynamical diffraction theory. ${ }^{39-41}$ Good agreement between the experimental and theoretical rocking curves (thin line in Fig. 3) could be achieved assuming that the $\mathrm{ZnSe}$ and AlAs layers grow pseudomorphically on GaAs substrate, i.e., for a parallel mismatch $f_{\|}=0$ for $\mathrm{ZnSe}$ and AlAs and perpendicular mismatch $f_{\perp}=5.9 \times 10^{-3}$ and 2.8 $\times 10^{-3}$ for $\mathrm{ZnSe}$ and AlAs, respectively.

This is confirmed by the reciprocal-space maps shown in Fig. 4, where the peaks originating from the GaAs substrate, and from the $\mathrm{ZnSe}$ and the AlAs layers are all located at the same $q_{x}$ value within our experimental resolution. In these maps, both the $\mathrm{ZnSe}$ and AlAs diffraction peaks are very narrow along the $q_{x}$ direction, with a full width at half maxi-

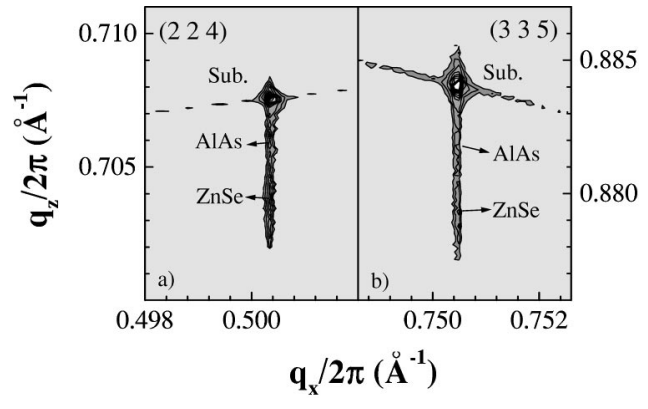

FIG. 4. Reciprocal space maps near the asymmetric (224) and (335) reflections for a heterostructure incorporating a 48-nm-thick ZnSe overlayer, a 36-nm-thick AlAs layer, and a 9-nm-thick $\mathrm{Al}_{0.3} \mathrm{Ga}_{0.7} \mathrm{As}$ buffer (Ref. 38). The contour levels are in steps of 0.3 in a logarithmic scale.

mum (FWHM) of about $7 \times 10^{-5} \AA^{-1}$. This value is close to the resolution of our diffractometer $\left(4 \times 10^{-5} \AA^{-1}\right)$, and thus reflects the high quality of both the $\mathrm{AlAs}$ and $\mathrm{ZnSe}$ layers.

Changes in interface composition did not affect the structural results discussed in this section. However, such changes do affect the stacking fault concentration, as also shown by previous studies of $\mathrm{ZnSe} / \mathrm{GaAs}$ heterostructures. ${ }^{27,32} \mathrm{~A}$ discussion of the systematic relation between interface composition and stacking fault density is, however, beyond the scope of this paper.

\section{B. Interface composition}

Information on the early stages of interface formation and the local interface composition was derived from XPS in situ. In particular, we systematically examined the integrated intensity and line shape of the photoelectron emission from the Al $2 p$, As $3 d$, $\mathrm{Zn} 3 d$, and Se $3 d$ core levels as a function of the $\mathrm{ZnSe}$ coverage $\vartheta$. The latter was calculated on the basis of growth rate calibrations.

In Fig. 5(a) we summarize, for example, results for the emission intensity from the $\mathrm{Al} 2 p$ and $\mathrm{Zn} 3 d$ doublets in the early stages of heterostructure fabrication with $\mathrm{BPR}=10$ (Zn-rich growth conditions, circles), and BPR $=0.1$ (Se-rich growth conditions, squares). In the plot we show in a logarithmic scale $I_{\mathrm{Al}}(\vartheta) / I_{\mathrm{Al}}^{0}$ (solid symbols) and 1 $-\left[I_{\mathrm{Zn}}(\vartheta) / I_{\mathrm{Zn}}^{\infty}\right]$ (open symbols), where $I$ denotes the integrated emission intensity after background subtraction and the subscript specifies the core level under consideration. The superscript denotes the initial emission intensity from the uncovered substrate or the emission intensity expected for an overlayer of infinite thickness.

In the absence of interdiffusion and reaction, and in the presence of layer-by-layer growth of the $\mathrm{ZnSe}$ overlayer, the functional dependence expected for the two data sets is identical:

$$
\ln \left[\frac{I_{\mathrm{Al}}(\vartheta)}{I_{\mathrm{Al}}^{0}}\right]=-\frac{\vartheta}{\lambda}=\ln \left[1-\frac{I_{\mathrm{Zn}}(\vartheta)}{I_{\mathrm{Zn}}^{\infty}}\right],
$$

where to the photoelectron escape depth $\lambda$ is the effective photoelectron escape depth. The four data sets in Fig. 5(a) 


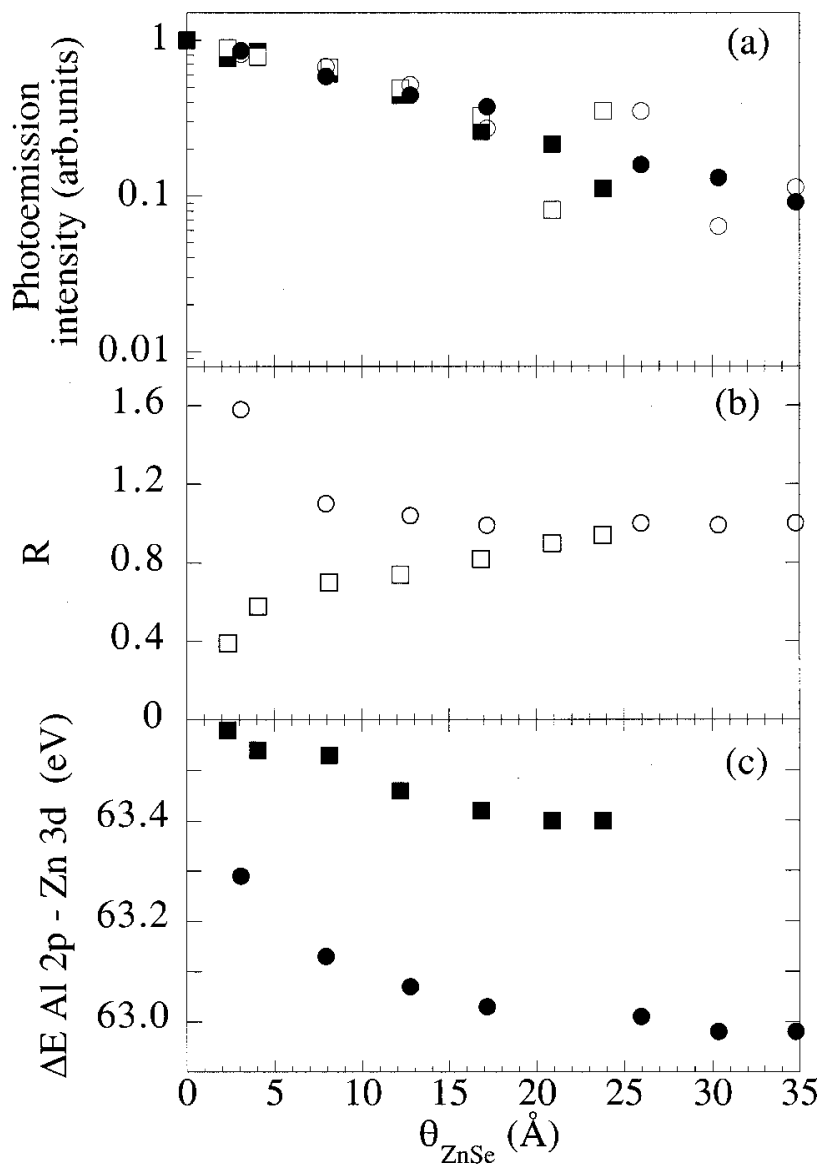

FIG. 5. (a) Emission intensity from the $\mathrm{Al} 2 p$ and $\mathrm{Zn} 3 d$ core doublets in the early stages of $\mathrm{ZnSe} / \mathrm{AlAs}$ heterostructure fabrication with $\mathrm{Zn} / \mathrm{Se}$ beam pressure ratio (BPR) of 10 (circles) and 0.1 (squares). In the plot we show $I_{\mathrm{Al}}(\vartheta) / I_{\mathrm{Al}}^{0}$ (solid symbols) and 1 $-\left[I_{\mathrm{Zn}}(\vartheta) / I_{\mathrm{Zn}}^{\infty}\right]$ (open symbols), where $I$ denotes the integrated emission intensity after background subtraction and the subscript specifies the core level under consideration. (b) Normalized $\mathrm{Zn}$ to Se photoemission intensity ratio $R_{\mathrm{ZnSe}}$ as a function of the $\mathrm{ZnSe}$ thickness during interface fabrication with $\mathrm{BPR}=10$ (open circles), and $\mathrm{BPR}=0.1$ (open squares). (c) $\mathrm{Zn} 3 d-\mathrm{Al} 2 p$ core-level separation as a function of $\mathrm{ZnSe}$ thickness, for $\mathrm{BPR}=10$ (solid circles), and $\mathrm{BPR}=0.1$ (solid squares).

are consistent with each other, within experimental uncertainty, and do follow the functional dependence described in Eq. (1). A least-squares fit of the data to Eq. (1) yields an experimental value of $\lambda$ of $1.4 \pm 0.1 \mathrm{~nm}$, also in good agreement with the expected photoelectron escape depth. ${ }^{28}$ The implication of the results of Fig. 5(a) is that, within the XPS experimental uncertainty, ZnSe grows layer by layer on AlAs with no evidence of atomic interdiffusion across the interface in growth conditions examined, for high as well as low BPR's.

Using the integrated emission intensity from the $\mathrm{Zn} 3 d$ and Se $3 d$ doublets after background subtraction, we calculated the XPS-derived $\mathrm{Zn} / \mathrm{Se}$ ratio as a function of $\mathrm{ZnSe}$ coverage, normalized to the value observed in all $\mathrm{ZnSe}$ bulk standards irrespective of the BPR:

$$
R_{\mathrm{Zn} / \mathrm{Se}}(\vartheta)=\frac{I_{\mathrm{Zn}}(\vartheta) / I_{\mathrm{Se}}(\vartheta)}{I_{\mathrm{Zn}}^{\infty} / I_{\mathrm{Se}}^{\infty}}
$$

In Fig. 5(b) we show the results obtained during interface fabrication with $\mathrm{BPR}=10$ (circles), and $\mathrm{BPR}=0.1$ (squares). Relatively large deviations of $R_{\text {ZnSe }}$ from unity are observed in the early growth stages. High $\mathrm{Zn}(\mathrm{Se})$ overpressure corresponds to Zn-rich (Se-rich) values of $R_{\mathrm{ZnSe}}$ in the early growth stage. The trend is compellingly similar to that observed earlier for $\mathrm{ZnSe} / \mathrm{GaAs}(001)$ heterostructures as a function of $\mathrm{Zn} / \mathrm{Se}$ BPR throughout the 0.1-10 range. ${ }^{4}$

We emphasize that any difference in the reconstructionrelated surface stoichiometry is per se quantitatively insufficient to explain the different coverage dependence of $R_{\mathrm{ZnSe}}$ for the different BPR's. The $2 \times 1$ surface reconstruction observed during $\mathrm{ZnSe}$ growth with $\mathrm{BPR}=0.1$ is believed to correspond to a surface terminated by a fully dimerized monolayer of $\mathrm{Se}^{42}$ while the $c(2 \times 2)$ reconstruction observed during $\mathrm{ZnSe}$ growth with $\mathrm{BPR}=10$ is believed to correspond to a surface terminated by half an ML of $\mathrm{Zn}$ atoms on a complete $\mathrm{ML}$ of $\mathrm{Se}$, i.e., to an ordered array of $\mathrm{Zn}$ vacancies within an outermost layer of $\mathrm{Zn}$ atoms. ${ }^{42-44}$ The different reconstructions would therefore also tend to give deviations of $R_{\mathrm{ZnSe}}$ from unity at low coverages.

The magnitude of such deviations can be roughly estimated along the lines proposed by Lazzarino et al. ${ }^{45}$ using the atomistic models just described for the $c(2 \times 2)$ and 2 $\times 1$ reconstructions, ${ }^{42-44}$ given the $\mathrm{Zn} 3 d$ emission intensity from a single (001) plane of $\mathrm{Zn}$ atoms $i_{\mathrm{Zn}}$, the Se $3 d$ emission intensity from a single (001) plane of Se atoms $i_{\mathrm{Se}}$, and using $1 \mathrm{ML}=0.1417 \mathrm{~nm}=d$ as the interplanar spacing in bulk ZnSe. ${ }^{45}$ For a $2 N$-monolayer-thick ZnSe layer, the expected $\mathrm{Zn} / \mathrm{Se}$ photoemission intensity ratio is given by $I_{\mathrm{Zn}} / I_{\mathrm{Se}}=\left(i_{\mathrm{Zn}} / i_{\mathrm{Se}}\right) e^{-d / \lambda}$ (i.e., independent of $\left.N\right)$ for a $2 \times 1$ reconstructed surface, and by

$$
\frac{I_{\mathrm{Zn}}}{I_{\mathrm{Se}}}(2 N)=\frac{i_{\mathrm{Zn}}}{i_{\mathrm{Se}}} \frac{\sum_{j=0}^{2 N} e^{-j d / \lambda}}{\sum_{j=0}^{2 N-1} e^{-j d / \lambda}}
$$

for a $c(2 \times 2)$ reconstructed surface. From these expressions, and their high coverage limit $I_{\mathrm{Zn}}^{\infty} / I_{\mathrm{Se}}^{\infty}$, one can evaluate ${ }^{46}$ the expected value of $R_{\mathrm{ZnSe}}$ in Eq. (2).

The calculated values of $R_{\mathrm{ZnSe}}$ at different nominal $\mathrm{ZnSe}$ coverages $(2,4,8 \mathrm{ML}$ and in the thick-overlayer limit) are summarized in Table I. We conclude that no more than $20 \%$ of the deviation from stoichiometry depicted in Fig. 5(b) can be explained by the different reconstruction-related surface stoichiometry. Therefore, a substantial Zn- or Se-enrichment of the interface region must result from employing Zn-rich or Se-rich growth conditions in the early stages of interface fabrication.

Information on the $\mathrm{Al}$ and $\mathrm{As}$ concentration in the interface region is more difficult to obtain. At XPS energies only minimal variations in the $\mathrm{Al} / \mathrm{As}$ ratio could be detected between interfaces fabricated with different BPR. This is not surprising, since the small interface contribution to the $\mathrm{Al}$ 
TABLE I. Calculated value of the $\mathrm{Zn} / \mathrm{Se}$ core photoemission intensity ratio $R_{\mathrm{Zn} / \mathrm{Se}}$ expected from a ZnSe epilayer with $c(2 \times 2)$ or $2 \times 1$ surface reconstruction at selected nominal coverages (Ref. 46). The values were calculated along the lines employed by Lazzarino et al. in Ref. 45, using the atomistic models for the $c$ (2 $\times 2$ ) and $2 \times 1$ reconstructions put forth by other authors in Refs. $42-44$.

\begin{tabular}{ccccc}
\hline \hline Reconstruction & $2 \mathrm{ML}$ & $4 \mathrm{ML}$ & $8 \mathrm{ML}$ & $\infty$ \\
\hline$c(2 \times 2)$ & 1.57 & 1.32 & 1.19 & 1.10 \\
$2 \times 1$ & 1 & 1 & 1 & 1 \\
\hline \hline
\end{tabular}

and As core emission is superimposed in all cases to the much larger signal from the underlying AlAs bulk. ${ }^{4}$

Recently Funato et al. have proposed a method to obtain from XPS data information on the $\mathrm{Ga} / \mathrm{As}$ ratio near $\mathrm{ZnSe} /$ GaAs interfaces. ${ }^{16}$ Starting from II-VI overlayers $3-5 \mathrm{~nm}$ thick, the authors monitored the core-level intensity ratios while the ZnSe layer thickness was reduced to subnanometer values by $\mathrm{Ar}^{+}$bombardment. The apparent changes in the depth dependence of the $\mathrm{Ga} / \mathrm{As}$ ratio from sample to sample were associated with differences in the III-V first-layer composition within abrupt II-VI/III-V interfaces. The author's argument was based on the assumption that the XPS sampling depth being finite, results for thick $(>3 \mathrm{~nm}) \mathrm{ZnSe}$ overlayers should be more sensitive to the interface region than results for thin ZnSe overlayers. ${ }^{16}$

Unfortunately, the above argument is incorrect. For an abrupt interface, in the presence of an exponential attenuation of the photoelectron emission as a function of depth, the core-level emission from the first III-V layer and from the residual III-V bulk will be attenuated in equal proportions by the II-VI overlayer. Consequently, the relative interface contribution to the overall III-V signal will remain constant while the II-VI thickness decreases. No change in the sensitivity to the interface composition is expected.

Therefore the reported changes in $\mathrm{Ga}$ /As ratio in heterojunctions grown in different MOCVD conditions ${ }^{16}$ cannot be explained by differences in the III-V first-layer composition within abrupt II-VI/III-V interfaces. It is more likely that the data reflect a different degree of $\mathrm{Ga}$ outdiffusion into the $\mathrm{ZnSe}$ overlayer. Depth profiling, in the absence of preferential sputtering artifacts, can provide information on atomic intermixing. Atomic intermixing might be enhanced by the relatively high growth temperatures employed in MOCVD $\left(450^{\circ} \mathrm{C}\right)$. When $\mathrm{ZnSe} / \mathrm{GaAs}$ heterojunctions grown by $\mathrm{MBE}$ at $290{ }^{\circ} \mathrm{C}$ are subsequently annealed above $400{ }^{\circ} \mathrm{C}$, interdiffusion leads to the formation of defect centers involving substitutional $\mathrm{Ga}$ atoms on $\mathrm{Zn}$ sites and $\mathrm{Zn}$ vacancies. ${ }^{47,48}$

We also changed the interface composition by dosing the $\operatorname{AlAs}(001) 3 \times 1$ surface with $\mathrm{Zn}$ or Se prior to $\mathrm{ZnSe}$ growth. Following GT predosing, XPS analysis of the Al $2 p$, As $3 d$, $\mathrm{Zn} 3 d$, and Se $3 d$ core-level intensity and comparison with the predictions of Eq. (1) revealed that only a relatively low saturation coverage could be obtained $(0.08-0.1 \mathrm{~nm}$ for $\mathrm{Zn}$ and about $0.15 \mathrm{~nm}$ for Se). Such coverages were not sufficient to induce large band offset modifications.

By lowering the substrate temperature down to RT we were able to deposit arbitrarily thick $\mathrm{Zn}$ or Se layers on AlAs. For our studies of the band offsets, we employed Se coverages of 1-3 ML and $\mathrm{Zn}$ coverages of $1 \mathrm{ML}$. Predosing was followed by $\mathrm{ZnSe}$ growth at $\mathrm{RT}$ with $\mathrm{BPR}=1$, i.e., with the same reconstruction and surface stoichiometry, on Zndosed and Se-dosed substrates. Information on the interface composition was also obtained from the normalized $\mathrm{Zn} / \mathrm{Se}$ photoemission intensity ratio $R_{\mathrm{ZnSe}}$. Values of $R_{\mathrm{ZnSe}}$ ranging between 1 and 0.7 were observed for Se-dosed surface while $R_{\mathrm{ZnSe}}$ values as high as 2.1 were obtained for the $\mathrm{Zn}$-dosed substrates. ${ }^{49}$ Such values, when compared with those in Fig. 5 (b), suggest that the two heterojunction fabrication methods both give rise to a substantial enhancement in the $\mathrm{Zn}$ or Se concentration near the interface. We caution the reader, however, that any similarity in the $R_{\mathrm{ZnSe}}$ values cannot rule out per se that the different fabrication procedures might lead to different composition profiles.

\section{Core-level positions and line shapes}

The chemical state of the excess $\mathrm{Zn}$ or Se near the interface is more difficult to probe by XPS, in view of the superposition of interface- and overlayer-related $\mathrm{Zn}$ and Se corelevel features and because of the limited experimental resolution available. The line shapes of both overlayer and substrate core levels was monitored as a function of overlayer thickness in the coverage range (1.5-2.5 nm) customarily employed for XPS determination of the band discontinuities. A constant line shape was observed for each core doublet throughout the above coverage range, irrespective of BPR and predosing.

In the same coverage range the $\mathrm{Zn} 3 d-\mathrm{Al} 2 p$ core level separation [Fig. 5(c)] as well as the Se $3 d-\mathrm{Al} 3 d$ core-level separation (not shown) were found to be independent of overlayer thickness, ruling out the presence of major chemically-shifted or surface-shifted contributions. Only in the case of $\mathrm{ZnSe} / \mathrm{AlAs}$ heterostructures fabricated on $\mathrm{Si}$ doped, $n^{+}$-type AlAs substrates, increased broadening of the Al $2 p$ and As $3 d$ core line shapes suggested the presence of relevant band-bending-related effects within the photoemission sampling depth.

In the leftmost section of Fig. 6 we show the line shape of the $\mathrm{Al} 2 p$ doublet for a clean, nominally undoped AlAs surface (a) and two $\mathrm{ZnSe} / \mathrm{AlAs}$ heterojunctions also fabricated on nominally undoped AlAs epilayers (b) and (c). The zero of the energy scale was taken at the position of the centroid of the $\mathrm{Al} 2 p$ emission from each interface. ${ }^{50}$ The three $\mathrm{Al} 2 p$ line shapes are identical, with an FWHM of $0.95 \mathrm{eV}$ in each case. The corresponding result for a $\mathrm{ZnSe} / \mathrm{AlAs}$ heterojunction fabricated on an $n^{+}$-type AlAs substrate with nominal doping of $1.5 \times 10^{18} \mathrm{~cm}^{-3}$ is shown in Fig. 7 (solid circles). A more asymmetric line shape with a FWHM of $1.15 \mathrm{eV}$ is observed. An analogous broadening was observed for the As $3 d$ doublet (not shown).

The broadened Al $2 p$ line shape in Fig. 7 could be reproduced by a superposition of individual contributions deriving from the individual atomic layers within the sampling depth. A least-squares fit to the data (solid line) was performed by 


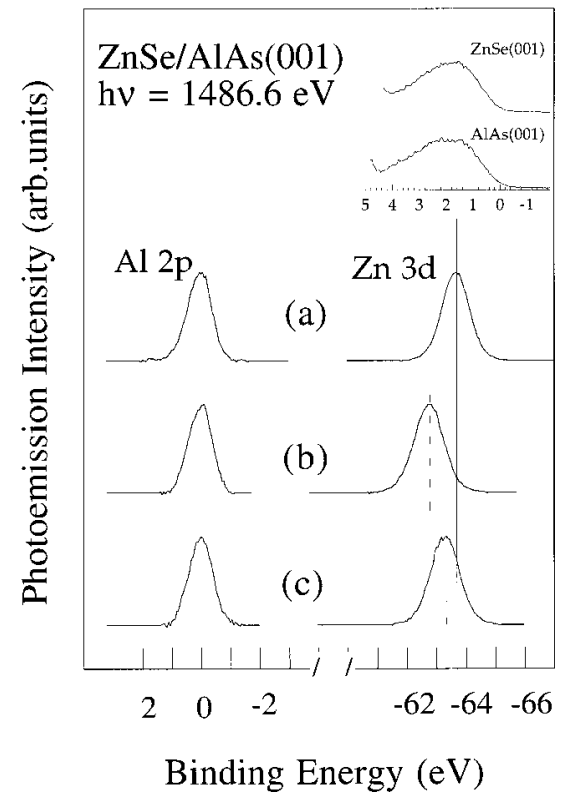

FIG. 6. Inset: Valence-band photoemission spectra from AlAs and $\mathrm{ZnSe}$ standards. (a) $\mathrm{Al} 2 p$ and $\mathrm{Zn} 3 d$ core level emission from the same standards. The binding energy of each centroid was measured relative to the valence-band maximum of each material. The separation between the core levels is therefore that expected from an hypothetical heterojunction with zero valence-band offset. (b) Core photoemission from a ZnSe/AlAs(001) heterojunction fabricated with $\mathrm{BPR}=10$. The $\mathrm{ZnSe}$ thickness was $2 \mathrm{~nm}$. (c) Core emission from a $\mathrm{ZnSe} / \mathrm{AlAs}(001)$ heterojunction fabricated with BPR $=0.4$. The ZnSe thickness was $2 \mathrm{~nm}$. The zero of the energy scale was taken at the position of the centroid of the $\mathrm{Al} 2 p$ doublet for each heterojunction (leftmost solid vertical line). The difference between the actual $\mathrm{Zn} 3 d$ position in (b) and (c) relative to (a) reflects valence-band offsets of $0.99 \pm 0.07$ and $0.40 \pm 0.07 \mathrm{eV}$, respectively.

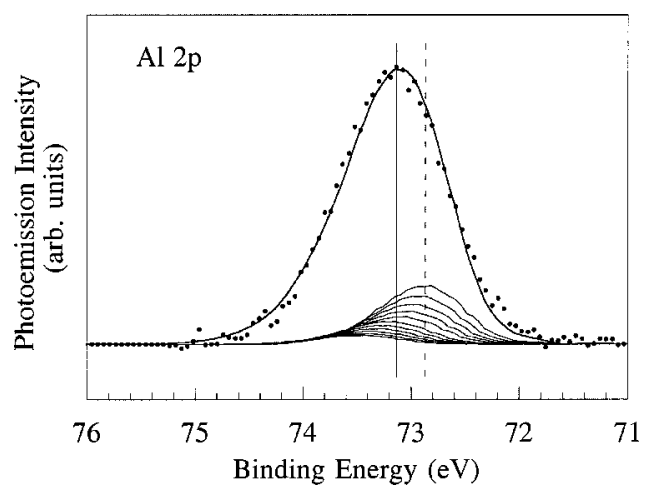

FIG. 7. Al $2 p$ core emission from a $n^{+}$-type AlAs epilayer (solid circles). The zero of the energy scale is taken at the position of the Fermi level of the sample. The broadened Al $2 p$ line shape could be reproduced by a superposition of individual contributions deriving from the individual atomic layers reflecting band bending within the sampling depth (solid line). The individual contributions from the ten topmost atomic layers are also shown (thin solid line). The measured Debye length indicates substantial Si segregation in the surface region. associating with each individual layer a phenomenological Al $2 p$ line shape of the type depicted in Fig. 6, with intensity attenuated by an escape-depth-determined exponential factor (thin solid line). A parabolic band-bending profile was postulated within the sampling depth, so that the only fitting parameters remaining were the Debye length within the XPS sampling depth, i.e., the dopant concentration in the nearsurface region $\left(7 \times 10^{18} \mathrm{~cm}^{-3}\right.$ in Fig. 7), and the interface position of the Fermi level ( $2.0 \mathrm{eV}$ above the AlAs valenceband maximum in Fig. 7).

Because of band bending, the apparent position of the centroid of the experimental $\mathrm{Al} 2 p$ doublet in Fig. 7 (vertical solid line) is $0.26 \mathrm{eV}$ below the position of the interfacerelated $\mathrm{Al} 2 p$ doublet (vertical dashed line). The value of the surface Debye length obtained from the fit in Fig. 6 indicates strong $\mathrm{Si}$ segregation during AlAs growth. Typically, Si concentrations at the surface were found to be 3-4 times higher than the nominal concentration for bulk doping levels in the low $10^{18}$ range and growth temperatures near $600^{\circ} \mathrm{C}$. This type of surface segregation, or surface riding, has been reported earlier for GaAs and $\mathrm{Al}_{x} \mathrm{Ga}_{1-x} \mathrm{As},{ }^{51}$ but we are not aware of any previous quantitative work on AlAs. The results of Fig. 7 were used to compensate for band-bending effects during band-offset measurements on highly doped substrates.

\section{Experimental band alignment}

The valence band discontinuities corresponding to the different interface compositions were determined in situ by XPS from the $\mathrm{Al} 2 p$ and $\mathrm{Zn} 3 d$ core-level energy separation in the 1.5-2.5 nm ZnSe coverage range. Identical results were obtained using the As $3 d$ and Se $3 d$ core separation, ruling out artifacts due to chemical shifts. Because of the small lattice mismatch between the different materials, the effect of pseudomorphic strain on the XPS determination of the offset can be neglected.

The procedure is illustrated in Fig. 6. In the inset we show valence-band spectra for an AlAs epitaxial substrate (bottom) and a 500-nm-thick ZnSe overlayer (top). The binding energy scale is referenced to the valence-band maximum $E_{\nu}$ as derived from a least-squares linear fit of the leading valence-band edge. ${ }^{52,53}$ Spectra for the $\mathrm{Al} 2 p$ and $\mathrm{Zn} 3 d$ core emission from these two samples are shown directly below the inset [Fig. 6(a)]. The core binding energies were measured relative to $E_{\nu}$ for each sample, and the zero of the energy scale was taken at the position of the $\mathrm{Al} 2 p$ centroid in AlAs. The corresponding core binding energy difference, $\Delta E_{b}=63.70 \pm 0.04 \mathrm{eV}$, is therefore that expected from a hypothetical heterojunction with zero valence-band offset.

Representative results for the core emission from $\mathrm{ZnSe} /$ $\mathrm{AlAs}$ interfaces fabricated with $\mathrm{BPR}=10$ and 0.4 are shown in Figs. 6(b) and 6(c), respectively. The ZnSe coverage was $2 \mathrm{~nm}$ in both cases. The zero of the energy scale was taken at the position of the $\mathrm{Al} 2 p$ centroid for each heterojunction. The corresponding interface core-level separation was $62.71 \pm 0.04$ and $63.30 \pm 0.04 \mathrm{eV}$ for heterojunctions fabricated in Zn-rich and Se-rich growth conditions, respectively, and was independent of overlayer thickness in the coverage 


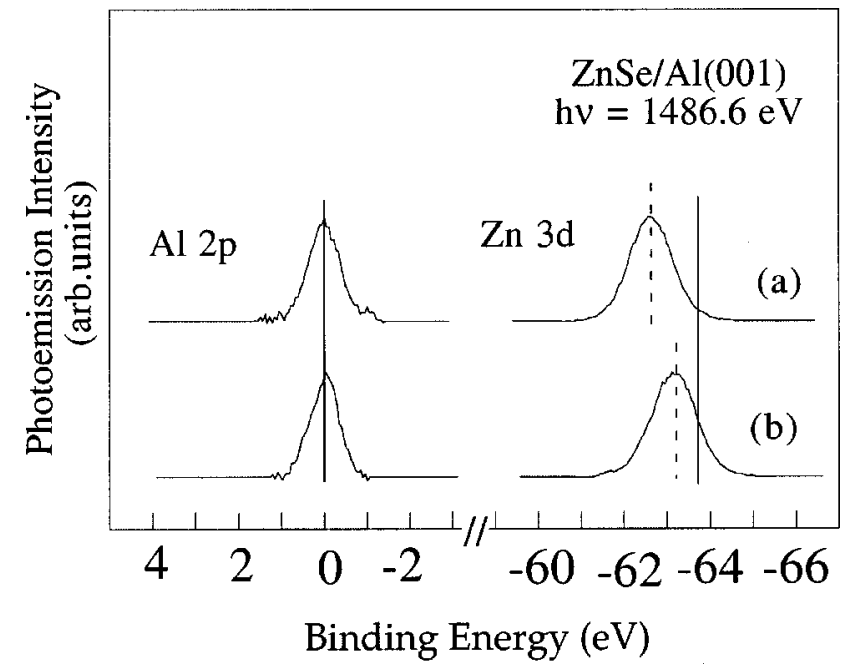

FIG. 8. $\mathrm{Al} 2 p$ and $\mathrm{Zn} 3 d$ core emission from $\mathrm{ZnSe} / \mathrm{AlAs}(001)$ heterojunctions fabricated after absorption of $1 \mathrm{ML}$ of $\mathrm{Zn}$ (a) and 2 ML of Se (b) onto AlAs(001) surfaces at room temperature. The $\mathrm{ZnSe}$ coverage was $2 \mathrm{~nm}$ in both cases. The zero of the energy scale was taken at the position of the centroid of the $\mathrm{Al} 2 p$ doublet for each heterojunction (leftmost solid vertical line). The position of the Zn $3 d$ centroid expected from a hypothetical heterojunction with zero valence-band offset is also shown (rightmost vertical line). For the two interfaces, we found $\Delta E_{v}=1.07 \pm 0.07$ and 0.56 $\pm 0.07 \mathrm{eV}$.

range of interest. The variation in the core-level separation relative to Fig. 5(a) gives directly the valence-band discontinuity. For the two interfaces in Fig. 6 we found $\Delta E_{\nu}=0.99$ \pm 0.07 and $0.40 \pm 0.07 \mathrm{eV}$, respectively, with the valenceband maximum of AlAs lying above that of $\mathrm{ZnSe}$ in both cases.

The same method of band offset determination was applied to heterostructures in which the interface composition was controlled through $\mathrm{Zn}$ or Se predosing. In Fig. 8 we show representative results for the core emission from $\mathrm{ZnSe} /$ AlAs heterojunctions fabricated after absorption of $1 \mathrm{ML}$ of $\mathrm{Zn}$ (a) and $2 \mathrm{ML}$ of Se (b) onto the AlAs(001) surface. The $\mathrm{ZnSe}$ coverage was $2 \mathrm{~nm}$ in both cases. The core line shapes for heterojunctions fabricated through Se/Zn predosing (Fig. 8) and low/high BPR (Fig. 6) are quantitatively consistent with one another. As in Fig. 6, in Fig. 8 the zero of the energy scale was taken at the position of the centroid of the Al $2 p$ doublet for each heterojunction (leftmost solid vertical line). The position of the $\mathrm{Zn} 3 d$ centroid expected from a hypothetical heterojunction with zero valence-band offset is shown by the rightmost vertical line. For the two interfaces in Fig. 8 we found $\Delta E_{\nu}=1.07 \pm 0.07$ and $0.56 \pm 0.07 \mathrm{eV}$, respectively, with the valence-band maximum of AlAs lying above that of $\mathrm{ZnSe}$ in both cases. ${ }^{54}$

The qualitative trend relating the band offsets to the localinterface composition is therefore essentially the same for heterojunctions fabricated through $\mathrm{Zn} / \mathrm{Se}$ predosing and high/low BPR. Zn-rich interface compositions correspond to relatively high values of the valence-band offset, while Serich interfaces exhibit substantially lower values of the valence-band discontinuity. A summary of all available ex-

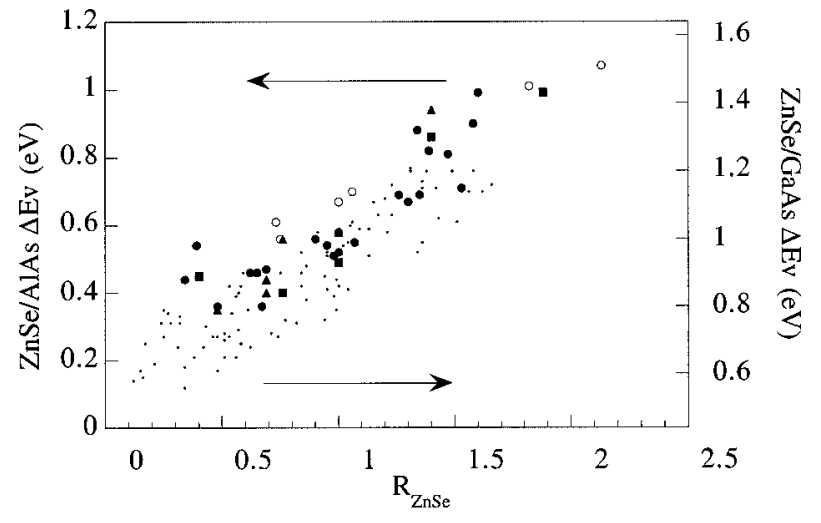

FIG. 9. Valence-band discontinuity (leftmost vertical scale) for $\mathrm{ZnSe/AlAs}(001)$ heterojunctions (large symbols) as a function of the value of $R_{\mathrm{ZnSe}}$ observed for each interface at a $\mathrm{ZnSe}$ coverage of $0.3 \mathrm{~nm}$. Different types of symbols denote results obtained for different AlAs doping levels and doping types (solid circles, $n$ $\sim 10^{18} \mathrm{~cm}^{-3}$; triangles, $p \sim 10^{16}-10^{18} \mathrm{~cm}^{-3}$, squares, undoped). Results for heterojunctions obtained through RT Se or Zn predosing and postgrowth annealing are also included (open circles). Earlier results for $\mathrm{ZnSe} / \mathrm{GaAs}$ heterojunctions (Ref. 4) are also shown for comparison (small symbols, rightmost vertical scale).

perimental data is shown in Fig. 9. Different solid symbols denote results obtained for different doping levels and doping types. Open symbols correspond to results for heterojunctions fabricated through $\mathrm{Zn}$ or Se predosing at RT and postgrowth annealing.

The valence-band offsets in Fig. 9 (leftmost vertical scale) were determined for overlayer coverages at which the $\mathrm{ZnSe}$ film appeared stoichiometric $(2-3 \mathrm{~nm})$, but are plotted versus the experimental value of the $\mathrm{Zn} / \mathrm{Se}$ ratio $R_{\mathrm{ZnSe}}$ observed for each interface in the early growth stage, i.e., at an arbitrary $\mathrm{ZnSe}$ coverage of $0.3 \mathrm{~nm}$. Maximum valence-band offsets in the 1.1-eV range are observed for interfaces grown with the highest BPR's explored (Zn-rich case), or for interfaces fabricated with $\mathrm{BPR}=1$ after $\mathrm{Zn}$ predosing. Much lower offsets (as low as $0.3-0.4 \mathrm{eV}$ ) were observed for the Se-rich case, or following Se predosing. The results in Fig. 9 indicate that the band alignment can be continuously tuned in a wide range of values, and that $R_{\mathrm{ZnSe}}$ can be used surprisingly well to parametrize the band-offset variation. Presumably, this is because $R_{\mathrm{ZnSe}}$ roughly gauges the local interface composition, that is fixed by the growth conditions employed in the early growth stages and which, in turns, determines the band discontinuities.

For comparison, we also show in Fig. 9 the corresponding results for $\mathrm{ZnSe} / \mathrm{GaAs}(001)$ heterojunctions (small symbols, rightmost vertical scale), also determined by XPS for interfaces grown with high/low $\mathrm{Zn} / \mathrm{Se} \mathrm{BPR} .{ }^{4}$ The zeros of the left and right vertical scales in Fig. 9 have been shifted relative to each other by $0.44 \mathrm{eV}$, i.e., by the average AlAs/ GaAs(001) valence-band offset. ${ }^{55}$ Such a shift brings the data sets for the two heterojunction systems in Fig. 9 in approximate superposition with each other. The implication is that, if similar values of the $R_{\mathrm{ZnSe}}$ parameter are considered for the two heterojunctions, the transitivity rule. ${ }^{1-3}$ is approximately verified. 


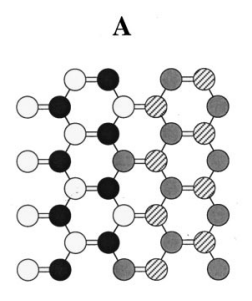

ZnSe-AlAs (+0.11 eV)

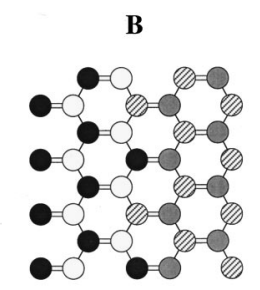

ZnSe-AlAs (+1.20 eV)

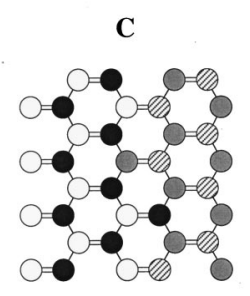

ZnSe-AlAs (+0.66 eV)

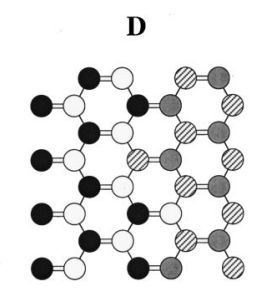

ZnSe-AlAs (+0.66 eV)

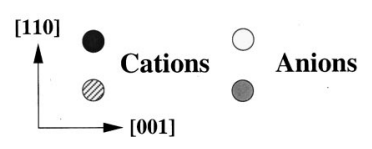

FIG. 10. Some of the simplest atomic configurations, for atomic intermixing on one or two atomic planes, that would give rise to neutral ZnSe-AlAs(001) interfaces. Below each configuration, we show the calculated value of the corresponding valence-band offset.

The predictions of the commutativity and transitivity rules for the band offsets have been calculated theoretically and found experimentally to hold for most isovalent heterojunctions. ${ }^{1-3}$ Deviations from such predictions are possible for heterovalent heterojunctions with polar orientation, though, since any of a number of inequivalent atomic configurations may be thermodynamically favored depending on the growth conditions. ${ }^{3}$ The wide offset tunability in Fig. 9, together with the verification of the prediction of the transitivity rule suggest that similar values of $R_{\mathrm{ZnSe}}$ for $\mathrm{ZnSe} / \mathrm{AlAs}$ and $\mathrm{ZnSe} / \mathrm{GaAs}$ must correspond to qualitatively similar interface configurations.

\section{E. Theoretical predictions}

Predictions of the band offsets presently require an assumption about which of the possible atomic configurations leading to neutral interfaces will actually form. Most authors have limited themselves to considering in their calculations some of the simplest configurations, ${ }^{3,4,14,15,56-58}$ with atomic mixing limited to one or two atomic planes. ${ }^{11,59,60}$

Four such configurations are schematically illustrated in Fig. 10 for $\mathrm{ZnSe}-\mathrm{AlAs}(001)$, viewed in the (110) plane. Configurations $A$ and $B$ correspond to mixed Se-As and $\mathrm{Zn}-\mathrm{Al}$ planes, respectively, with 50-50 composition at the interface. Configuration $C$ includes two mixed planes in sequence with Se-As 25-75 and $\mathrm{Zn}-\mathrm{Al}$ 75-25 compositions, respectively, between the Al-terminated AlAs substrate and

the Se-terminated $\mathrm{ZnSe}$ overlayer. Configuration $D$ is the complementary configuration, with $\mathrm{Zn}-\mathrm{Al} 25-75$ and Se-As 75-25 mixed planes between the As-terminated AlAs substrate and the $\mathrm{Zn}$-terminated $\mathrm{ZnSe}$ overlayer.

Configurations $A$ and $B$ are expected to correspond to minimum and maximum values for the band offsets, respectively. This reflects ionic dipoles of equal, maximum magnitude - for the type of intermixing envisioned-and opposite orientation. Configurations $C$ and $D$ correspond to no ionic dipole, as would be the case for abrupt, nonpolar (110) interfaces. First-principles calculations of the band offsets for $\mathrm{ZnSe} / \mathrm{GaAs}$ heterojunctions with configurations of the type depicted in Fig. 10 have produced results in good agreement with LRT predictions. ${ }^{4,14,15}$

We derived new predictions for the band discontinuities in $\mathrm{ZnSe} / \mathrm{AlAs}(001)$ heterojunctions using LRT arguments and the available first-principles results for $\mathrm{ZnSe} / \mathrm{GaAs}$. It has been shown that the valence-band offset can be expressed as the sum of two terms,

$$
\Delta E_{\nu}=\Delta E_{\nu}^{\mathrm{BS}}+\Delta V,
$$

where $\Delta E_{\nu}^{\mathrm{BS}}$, the band structure term, is the difference between the two valence-band maxima measured relative to the average electrostatic potential of each of the two semiconductors, and $\Delta V$ is the electrostatic potential energy lineup across the interface. The first term is a property of the bulk materials, and will satisfy the predictions of the transitivity rule, while the second term may depend on the charge distribution across the interface. ${ }^{12,14,58}$

The term $\Delta V$ depends on the relatively small difference between the charge distributions of the two bulks and can be calculated using perturbation theory. In the LRT framework, the interface can be viewed as a perturbation that changes the virtual ions of a periodic, zincblende virtual crystal into the real ions. The sum of the localized responses to such individual perturbations determines the potential energy lineup through Poisson's equation. ${ }^{58}$ At heterovalent interfaces $\Delta V$ can be expressed as the sum of two terms,

$$
\Delta V=\Delta V_{\text {iso }}+\Delta V_{\text {het }},
$$

where the first term comes from the neutral part of the perturbation, and the second term from the charged part. The first term is also independent of the details of the interface, while the second term is equivalent to the potential lineup generated by an assembly of point charges of absolute value $\Delta Z|e| / 2\langle\varepsilon\rangle$, where $\Delta Z$ is the charge of the localized perturbation and $\langle\varepsilon\rangle$ the average dielectric constant of materials 1 and 2 comprising the junction, expressed by ${ }^{12}$

$$
\langle\varepsilon\rangle=2\left(\frac{1}{\varepsilon_{1}}+\frac{1}{\varepsilon_{2}}\right)^{-1} .
$$

We determined the overall bulk-related contribution $\Delta E_{\nu}^{\mathrm{Bulk}}=\Delta E_{\nu}^{\mathrm{BS}}+\Delta V_{\mathrm{iso}}$, which is intrinsically transitive, from the available LRT results for $\mathrm{ZnSe} / \mathrm{GaAs}$ (1.11 eV, Ref. 4) and GaAs/AlAs ( $-0.45 \mathrm{eV}$, Ref. 12), and obtained $\Delta E_{\nu}^{\text {Bulk }}=0.66 \mathrm{eV}$ for ZnSe/AlAs. Within LRT this is the valence-band discontinuity expected for configurations $C$ 
and $D$, which have no ionic dipole, as well as for the abrupt, nonpolar (110)-oriented interface.,

We calculated the interface-specific $\Delta V_{\text {het }}$ terms for configurations $A$ and $B$ using Eq. (5) to rescale the earlier selfconsistent results for $\mathrm{ZnSe} / \mathrm{GaAs}(001)$. In Ref. 4 we found $\Delta V_{\text {het }}=-0.49$ and $+0.48 \mathrm{eV}$ for configurations $A$ and $B$, respectively. Using the appropriate dielectric screening for ZnSe/AlAs (Ref. 61) we found $\Delta V_{\text {het }}=-0.52$ and $+0.54 \mathrm{eV}$ for the two homologous configurations. For the overall valence-band offset we therefore obtained $\Delta E_{\nu}=\Delta E_{\nu}^{\text {Bulk }}$ $+\Delta V_{\text {het }}=+0.11$ and $+1.20 \mathrm{eV}$ for the Se-As and $\mathrm{Zn}-\mathrm{Al}$ mixed interface configurations $A$ and $B$, respectively. The numerical results obtained for each of the four model interface configurations have been included in Fig. 10.

We emphasize that the configurations in Fig. 10 correspond to only four of the simplest possible interface terminations that may lead to neutral interface. Nevertheless, the corresponding theoretical predictions seem to capture a number of important trends observed in the experimental systematics. Theory clearly indicates that a number of possible band discontinuities should exist, and that the offset tunability range should be wider for $\mathrm{ZnSe} / \mathrm{AlAs}$ than for $\mathrm{ZnSe} / \mathrm{GaAs}$, because of the reduced dielectric screening of the interface dipole. Both trends are observed in Fig. 10.

Predictions that a Se-As mixed interface should exhibit a low valence-band discontinuity, and a $\mathrm{Zn}-\mathrm{Al}$ interface a comparatively high offset are also suggestive, in view of the experimental fact that Se-rich interface terminations lead to lower valence-band offsets as compared to Zn-rich interfaces. Unfortunately, in the absence of reliable information about the $\mathrm{Ga} / \mathrm{As}$ ratio at the interface, we cannot probe the analogy any farther.

Finally, the experimental trend in Fig. 9 supporting the transitivity of the band offsets when heterojunctions with similar $R_{\mathrm{ZnSe}}(\vartheta)$ values are compared, finds a natural explanation in the LRT arguments that we used to extract the theoretical results in Fig. 10. Transitivity of the band offsets is expected even for heterovalent heterojunctions if the same types of interface configurations are considered, when strain and lattice relaxation play a negligible role, as in the present case.

\section{CONCLUSIONS}

We performed experimental and theoretical investigations of $\mathrm{ZnSe} / \mathrm{AlAs}$ heterostructures with (001) orientation. The goal was to probe the systematic relations between growth parameters, interface composition, and experimental band discontinuities as compared to the current theoretical predictions for the band alignment in heterovalent heterojunctions.

Epitaxial heterostructures of high crystalline quality were fabricated by MBE on AlAs $/ \mathrm{Al}_{x} \mathrm{Ga}_{1-x}$ As buffer layers and GaAs wafers. Throughout the pseudomorphic thickness range the only type of native extended defects observed in the heterostructures were stacking faults on $\{111\}$ planes, nucleating at the II-VI/III-V interface and propagating to the free surface of the II-VI overlayer.

The local interface composition was varied by adsorbing $\mathrm{Zn}$ or Se onto the $\mathrm{AlAs}(001)$ surface prior to $\mathrm{ZnSe}$ growth in stoichiometric growth conditions, or by employing Zn-rich or Se-rich growth conditions during the early stages of interface formation. The two methods yielded similar variations in the local $\mathrm{Zn} / \mathrm{Se}$ ratio at the interface, as determined by XPS.

The local $\mathrm{Zn} / \mathrm{Se}$ ratio was found to control the band alignment. Heterojunctions synthesized after Se adsorption or in Se-rich growth conditions exhibited comparatively low valence-band offsets (as low as $0.3-0.4 \mathrm{eV}$ ), while interfaces fabricated following $\mathrm{Zn}$ adsorption or in $\mathrm{Zn}$-rich growth conditions showed relatively high valence-band discontinuities (as high as $1.1 \mathrm{eV}$ ).

Furthermore, if results for $\mathrm{ZnSe} / \mathrm{AlAs}(001)$ heterojunctions are compared with those obtained from $\mathrm{ZnSe} /$ $\mathrm{GaAs}(001)$ interfaces with similar values of the local $\mathrm{Zn} / \mathrm{Se}$ ratio, the predictions of the transitivity rule are approximately verified.

Theoretical predictions for model interface configurations capture most of the essential experimental trends. In particular, they indicate that a number of possible band discontinuities may exist and that $\mathrm{Zn}$ - and Se-rich interface compositions might yield different offsets. The wider offset tunability range for $\mathrm{ZnSe} / \mathrm{AlAs}$ relative to $\mathrm{ZnSe} / \mathrm{GaAs}$ is also predicted, because of the reduced dielectric screening of the interface dipole. Finally, transitivity of the band offsets is expected even for heterovalent heterojunctions with polar orientation if the same types of interface configurations are considered, and if strain and lattice relaxation play a negligible role.

\section{ACKNOWLEDGMENTS}

This work was supported in part by the Consiglio Nazionale delle Ricerche under the MADESS project, and by the National Science Foundation under Grant No. DMR9819659. We thank N. Binggeli and M. Peressi for useful discussions or for providing us with copy of their work prior to publication.

\footnotetext{
*Also with Dipartimento di Fisica, Università di Modena e Reggio Emilia, I-41100 Modena, Italy.

†Also with Dipartimento di Fisica, Universita' di Trieste, I-34127 Trieste, Italy.

${ }^{1}$ G. Margaritondo and P. Perfetti, in Heterojunction Band Discontinuities: Physics and Device Applications, edited by F. Capasso and G. Margaritondo (North-Holland, Amsterdam, 1987), and references therein.

${ }^{2}$ L.J. Brillson, in Handbook on Semiconductors, edited by P.T.
}

Landsberg (North-Holland, Amsterdam, 1992), Vol. I, p. 281, and references therein.

${ }^{3}$ A. Franciosi and C.G. Van de Walle, Surf. Sci. Rep. 25, 1 (1996), and references therein.

${ }^{4}$ R. Nicolini, L. Vanzetti, Guido Mula, G. Bratina, L. Sorba, A. Franciosi, M. Peressi, S. Baroni, R. Resta, A. Baldereschi, J.E. Angelo, and W.W. Gerberich, Phys. Rev. Lett. 72, 294 (1994).

${ }^{5}$ A. Bonanni, L. Vanzetti, L. Sorba, A. Franciosi, M. Lomascolo, P. Prete, and R. Cingolani, Appl. Phys. Lett. 66, 1092 (1995). 
${ }^{6}$ G. Bratina, T. Ozzello, and A. Franciosi, J. Vac. Sci. Technol. B 14, 2967 (1996).

${ }^{7}$ G. Bratina, T. Ozzello, and A. Franciosi, J. Vac. Sci. Technol. A 14, 3135 (1996).

${ }^{8}$ X. Yang, L.J. Brillson, A.D. Raisanen, L. Vanzetti, A. Bonanni, and A. Franciosi, J. Vac. Sci. Technol. A 14, 867 (1996).

${ }^{9}$ V. Pellegrini, M. Börger, M. Lazzeri, F. Beltram, J.J. Paggel, L. Sorba, S. Rubini, M. Lazzarino, A. Franciosi, J.-M. Bonard, and J.-O. Ganiere, Appl. Phys. Lett. 69, 3233 (1996).

${ }^{10}$ M. Lazzeri, V. Pellegrini, F. Beltram, M. Lazzarino, J.J. Paggel, L. Sorba, S. Rubini, A. Bonanni, and A. Franciosi, J. Cryst. Growth 175-176, 603 (1997).

${ }^{11}$ W.A. Harrison, E.A. Kraut and J.R. Waldrop, and R.W. Grant, Phys. Rev. B 18, 4402 (1978); W.A. Harrison, J. Vac. Sci. Technol. 16, 1492 (1979).

${ }^{12} \mathrm{~S}$. Baroni, R. Resta, and A. Baldereschi, in Proceedings of the 19th International Conference on the Physics of Semiconductors, edited by W. Zawadzki (Institute of Physics, Polish Academy of Science, Wroclaw, 1988), p. 525; S. Baroni, R. Resta, A. Baldereschi, and M. Peressi, in Spectroscopy of Semiconductor Microstructures, edited by G. Fasol, A. Fasolino, and P. Lugli (Plenum, London, 1989). We caution the reader that Eq. (5) is shown without the required factor of two in the above references due to a typographical error.

${ }^{13}$ A. Baldereschi, S. Baroni, and R. Resta, Phys. Rev. Lett. 61, 734 (1988).

${ }^{14}$ M. Peressi, N. Binggeli, and A. Baldereschi, J. Phys. D 31, 1273 (1998), and references therein.

${ }^{15}$ A. Kley and J. Neugebauer, Phys. Rev. B 50, 8616 (1994).

${ }^{16}$ M. Funato, S. Aoki, S. Fujita, and S. Fujita, J. Appl. Phys. 82, 2984 (1997).

${ }^{17}$ M. Nagelstrasser, H. Dröge, F. Fischer, T. Litz, A. Waag, G. Landwehr, and H.-P. Steinrück, J. Appl. Phys. 83, 4253 (1998).

${ }^{18}$ T. Walter, A. Rosenauer, R. Wittmann, D. Gerthsen, F. Fischer, T. Gerhard, A. Waag, G. Landwehr, P. Schunk, and T. Schimmel, Phys. Rev. B 59, 8114 (1999).

${ }^{19}$ P. Perfetti, C. Quaresima, C. Coluzza, C. Fortunato, and G. Margaritondo, Phys. Rev. Lett. 57, 2065 (1986).

${ }^{20}$ B.J. Skromme, M.C. Tamargo, J.L. de Miguel, and R.E. Nahory, Appl. Phys. Lett. 53, 2217 (1988).

${ }^{21}$ S. Rubini, E. Milocco, L. Sorba, and A. Franciosi, J. Cryst. Growth 184-185, 178 (1998).

${ }^{22}$ W.I. Wang, J. Vac. Sci. Technol. B 1, 574 (1983); A.M. Dabiran and P.I. Cohen, J. Cryst. Growth 150, 23 (1995); R.W. Bernstein, A. Borg, H. Husby, B.-O. Fimland, and J.K. Grepstad, Appl. Surf. Sci. 56-58, 74 (1992).

${ }^{23}$ T. Yao and T. Takeda, Appl. Phys. Lett. 48, 160 (1986).

${ }^{24}$ M.C. Tamargo, J.L. de Miguel, D.M. Hwang, and H.H. Farrel, J. Vac. Sci. Technol. B 6, 784 (1988).

${ }^{25}$ L. Vanzetti, A. Bonanni, L. Sorba, A. Franciosi, M. Lomascolo, D. Greco, and R. Cingolani, in Proceedings 22th International Conference on the Physics of Semiconductors, edited by D.J. Lockwood (World Scientific, Singapore, 1995), pp. 767-770.

${ }^{26}$ G. Bratina, L. Vanzetti, A. Bonanni, L. Sorba, J.J. Paggel, A. Franciosi, T. Peluso, and L. Tapfer, J. Cryst. Growth 159, 703 (1996).

${ }^{27}$ S. Heun, J.J. Paggel, L. Sorba, S. Rubini, A. Franciosi, J.-M. Bonard, and J.-D. Ganiere, Appl. Phys. Lett. 70, 237 (1997).

${ }^{28}$ In the kinetic energy range of interest for the present study, E.A.
Kraut, R.W. Grant, J.R. Waldrop, and S.P. Kowalczyk, Phys. Rev. B 28, 1965 (1983), determined an inelastic mean free path of $2.66 \mathrm{~nm}$ for normally emitted photoelectrons. In our experimental geometry the average photoelectron collection angle is $55^{\circ}$ from the sample normal, leading to an effective escape depth of about $1.5 \mathrm{~nm}$.

${ }^{29}$ See, for example, P.F. Fewster, Semicond. Sci. Technol. 8, 1915 (1993).

${ }^{30}$ S. Guha, J.M. DePuydt, J. Qiu, G.E. Höfler, M.A. Haase, B.J. Wu, and H. Cheng, Appl. Phys. Lett. 63, 3023 (1993); S. Guha, J.M. DePuydt, M.A. Haase, J. Qiu, and H. Cheng, ibid. 63, 3107 (1993).

${ }^{31}$ J.-M. Bonard, J.-D. Ganiere, S. Heun, J.J. Paggel, S. Rubini, L. Sorba, and A. Franciosi, Philos. Mag. Lett. 75, 219 (1997), and references therein.

${ }^{32}$ S. Heun, J.J. Paggel, S. Rubini, and A. Franciosi, J. Vac. Sci. Technol. B 14, 2980 (1996).

${ }^{33}$ S. Miwa, L.H. Kuo, K. Kimura, T. Yasuda, A. Ohtake, C.G. Jin, and T. Yao, Appl. Phys. Lett. 73, 939 (1998).

${ }^{34}$ J.L. Batstone, J.W. Steeds, and P.J. Wright, Philos. Mag. 66, 609 (1992).

${ }^{35}$ S. Heun, J.J. Paggel, L. Sorba, S. Rubini, A. Franciosi, J.-M. Bonard, and J.-D. Ganiere, J. Vac. Sci. Technol. B 15, 1279 (1997).

${ }^{36}$ L.H. Kuo, K. Kimura, T. Yasuda, S. Miwa, C.G. Jin, K. Tanaka, and T. Yao, Appl. Phys. Lett. 68, 2413 (1996).

${ }^{37}$ In-plane and perpendicular lattice strain and mismatch are defined here as follows:

$$
\begin{gathered}
\varepsilon_{\|}=\frac{a_{\|}-a_{r}}{a_{r}}, \quad \varepsilon_{\perp}=\frac{a_{\perp}-a_{r}}{a_{r}}, \\
f_{\|}=\frac{a_{\|}-a_{0}}{a_{0}}, \quad f_{\perp}=\frac{a_{\perp}-a_{0}}{a_{0}},
\end{gathered}
$$

where $a_{\|}$and $a_{\perp}$ are the measured, strained lattice constants of the overlayer, and $a_{r}$ and $a_{0}$ are the relaxed lattice constant of the overlayer material and the lattice constant of the underlying GaAs buffer layer, respectively.

${ }^{38}$ For the samples examined we obtained from XRD a value of 46.3 $\mathrm{nm}$ for the thickness of ZnSe overlayer, $39.4 \mathrm{~nm}$ for the thickness of the AlAs layer, and $9 \mathrm{~nm}$ for the thickness of the $\mathrm{Al}_{0.3} \mathrm{Ga}_{0.7} \mathrm{As}$ buffer. In the calculations, we assumed that the $\mathrm{ZnSe}$ and AlAs layers were grown pseudomorphically onto the GaAs substrate, i.e., parallel mismatch $f_{\|}=0$ for both $\mathrm{ZnSe}$ and AlAs, and perpendicular mismatch $f_{\perp}=5.45 \times 10^{-3}$ and 2.77 $\times 10^{-3}$, respectively, for $\mathrm{ZnSe}$ and AlAs. The corresponding TEM-derived parameters for the same samples were $48 \pm 4 \mathrm{~nm}$ for the $\mathrm{ZnSe}$ overlayer, $36 \pm 3 \mathrm{~nm}$ for the AlAs layer, and 9 $\pm 1 \mathrm{~nm}$ for the $\mathrm{Al}_{0.3} \mathrm{Ga}_{0.7} \mathrm{As}$ buffer.

${ }^{39}$ L. Tapfer, L. De Caro, C. Giannini, H.-P. Schönherr, and K.H. Ploog, Solid State Commun. 98, 599 (1996).

${ }^{40}$ L. De Caro, C. Giannini, and L. Tapfer, J. Appl. Phys. 79, 4101 (1996).

${ }^{41}$ For the lattice constants the following values were used: $a^{\text {GaAs }}$ $=5.6535 \AA, a^{\mathrm{AlAs}}=5.6617 \AA$, and $a^{\mathrm{ZnSe}}=5.6689 \AA$, and for the elastic constants $c_{11}^{\mathrm{AlAs}}=119.9 \mathrm{GPa}, c_{12}^{\mathrm{AlAs}}=57.5 \mathrm{GPa}$, and $c_{11}^{\mathrm{ZnSe}}$ $=90.0 \mathrm{GPa}, c_{12}^{\mathrm{ZnSe}}=53.4 \mathrm{GPa}$.

${ }^{42}$ W. Chen, A. Kahn, P. Soukiassan, P.S. Mangat, J. Gaines, C. Ponzoni, and D. Olego, Phys. Rev. B 49, 10790 (1994). 
${ }^{43}$ M. Vos, F. Xu, S.G. Anderson, J.H. Weaver, and H. Cheng, Phys. Rev. B 39, 10744 (1989).

${ }^{44}$ H.H. Farrell, M.C. Tamargo, and S.M. Shibly, J. Vac. Sci. Technol. B 8, 884 (1990).

${ }^{45}$ M. Lazzarino, G. Scarel, S. Rubini, G. Bratina, L. Sorba, A. Franciosi, C. Berthod, N. Binggeli, and A. Baldereschi, Phys. Rev. B 57, R9431 (1998). With the convention employed, a ZnSe overlayer with nominal coverage $2 N$ corresponds to $N$ atomic layers of $\mathrm{Se}$ and $N$ atomic layers of $\mathrm{Zn}$, with the outermost layer being a full Se monolayer for the $2 \times 1$ surface termination, or an additional half monolayer of $\mathrm{Zn}$ atoms on top of the outermost Se atoms for the $c(2 \times 2)$ surface termination.

${ }^{46} \mathrm{We}$ caution the reader that, within the rough approximation employed, the high-coverage limits of the ZnSe intensity ratios are expected to be different for the $c(2 \times 2)$ and $2 \times 1$ reconstructions. In particular, for the $2 \times 1$ surface one expects $I_{\mathrm{Zn}}^{\infty} / I_{\mathrm{Se}}^{\infty}$ $=\left(i_{\mathrm{Zn}} / i_{\mathrm{Se}}\right) e^{-d / \lambda}$, while for $c(2 \times 2)$ surface it should be $I_{\mathrm{Zn}}^{\infty} / I_{\mathrm{Se}}^{\infty}=i_{\mathrm{Zn}} / i_{\mathrm{Se}}$. The two high-coverage limits differ by $10 \%$. To simplify comparison with Fig. 5(b) and estimate the maximum variations in $R_{\mathrm{ZnSe}}$, we have used the high-coverage limit of the $2 \times 1$ case to calculate $R_{\mathrm{ZnSe}}$ for both surfaces from Eq. (2).

${ }^{47}$ X. Yang, L.J. Brillson, A.D. Raisanen, L. Vanzetti, A. Bonanni, A. Franciosi, M. Grundmann, and D. Bimberg, J. Vac. Sci. Technol. B 14, 2961 (1996).

${ }^{48}$ A. Raisanen, L.J. Brillson, L. Vanzetti, L. Sorba, and A. Franciosi, Appl. Phys. Lett. 66, 3301 (1995).

${ }^{49}$ We emphasize that the $R_{\text {ZnSe }}$ values given above were measured on as-grown, 0.3-nm-thick ZnSe layers. As mentioned in Sec. II, RT-grown ZnSe was characterized by a polycrystalline-type of RHEED pattern. A low-binding-energy component was detected in the $\mathrm{Zn} 3 d$ core-level emission spectra for RT-deposited $\mathrm{ZnSe}$ layers thicker than $1 \mathrm{~nm}$, irrespective of the dosing procedure, suggesting the presence of segregated metallic Zn. Post-growth annealing at $290{ }^{\circ} \mathrm{C}$, in addition to improving crystal quality as indicated by RHEED, eliminated the extra component in the $\mathrm{Zn}$ $3 d$ line shape, and reduced the $R_{\mathrm{ZnSe}}$ values. The quoted $R_{\mathrm{ZnSe}}$ values are therefore to be considered only as upper limits, involving not only the interface-related contribution as in the case of low/high BPR experiments, but also the segregated $\mathrm{Zn}$ contribution.

${ }^{50}$ A spurious feature related to a plasmon replica of the Se $3 d$ line has been subtracted from the raw data in the $0-4 \mathrm{eV}$ range in the figure. The spurious emission was found to have an FWHM of $1.5 \mathrm{eV}$, and a binding energy $21 \mathrm{eV}$ higher than the Se $3 d$ line.

${ }^{51}$ O. Brandt, G.E. Crook, K. Ploog, J. Wagner, and M. Mayer, Appl. Phys. Lett. 59, 2732 (1991).

${ }^{52}$ See G. Bratina, R. Nicolini, L. Sorba, L. Vanzetti, Guido Mula, X. Yu, and A. Franciosi, J. Cryst. Growth 127, 387 (1993). In this reference, and in the present work, the position of the $\mathrm{Ga} 3 d$ and $\mathrm{Zn} 3 d$ core levels in GaAs and $\mathrm{ZnSe}$ bulk standards was referred to the valence-band maximum using a least-squares linear fit of the leading valence-band edge. Fits to a broadened theoretical density of states (obtained from nonlocal pseudopotential calculations) yielded consistent results.

${ }^{53}$ L. Sorba, G. Bratina, A. Antonini, A. Franciosi, L. Tapfer,A. Migliori, and P. Merli, Phys. Rev. B 46, 6834 (1992).

${ }^{54}$ Valence-band offset measurements on heterojunctions in which the predosing had been performed at GT yielded between 0.50 and $0.59 \pm 0.07 \mathrm{eV}$ for $\mathrm{Zn}$ predosing and between 0.44 and $0.49 \pm 0.07 \mathrm{eV}$ for Se predosing. The implication is that the relatively low saturation coverages that could be obtained using GT predosing were insufficient to achieve large band-offset modifications.

${ }^{55}$ Valence-band offsets of $0.42 \pm 0.03 \mathrm{eV}$ and $0.46 \pm 0.03 \mathrm{eV}$ were measured for AlAs/GaAs(001) and GaAs/AlAs(001) interfaces, respectively (Ref. 53). Such values are consistent with the results of several other determinations of the band offsets. See Refs. 1-3 for a survey of the literature.

${ }^{56}$ R.G. Dandrea, S. Froyen, and A. Zunger, Phys. Rev. B 42, 3213 (1990).

${ }^{57}$ G. Biasiol, L. Sorba, G. Bratina, R. Nicolini, A. Franciosi, M. Peressi, S. Baroni, R. Resta, and A. Baldereschi, Phys. Rev. Lett. 69, 1283 (1992).

${ }^{58}$ G. Bratina, L. Vanzetti, L. Sorba, G. Biasiol, A. Franciosi, M. Peressi, and S. Baroni, Phys. Rev. B 50, 11723 (1994).

${ }^{59}$ R.M. Martin, J. Vac. Sci. Technol. 17, 978 (1980).

${ }^{60}$ K. Kunc and R.M. Martin, Phys. Rev. B 24, 3445 (1981).

${ }^{61}$ To evaluate the interface dielectric screening by means of Eq. 5 we used $\varepsilon(\mathrm{ZnSe})=8.8$ from H.E. Gumlich, D. Theis, and D. Tschierse, in Numerical Data and Functional Relationship in Science and Technology, edited by O. Madelung, Landolt Boernstein, New Series, Group III, Vol. 17, Pt a (Springer, Berlin, 1982); $\varepsilon(\mathrm{GaAs})=13.18$ from G.A. Samara, Phys. Rev. B 27, 3494 (1983); and $\varepsilon(\mathrm{AlAs})=10.06$ from R.E. Fern and A. Onton, J. Appl. Phys. 42, 3499 (1971). 